\title{
Imaging Labeled Protein Complex Subunits in Whole Eukaryotic Cells in their Native Aqueous Environment
}

\author{
Niels de Jonge ${ }^{1,2}$ and Diana B. Peckys ${ }^{1}$ \\ 1. INM - Leibniz Institute for New Materials, Saarbrücken, Germany. \\ 2. Department of Molecular Physiology and Biophysics, Vanderbilt University School of Medicine, \\ Nashville, TN, USA
}

Cellular function is governed by the molecular machinery of cellular components, i.e., proteins, DNA, lipids, etc., dynamically assembling into functional structures such as protein- and other macromolecular complexes. Many scientific questions about the interplay of these structures relating to cellular function are not yet fully understood partly because methods are lacking to image them within the context of intact cells in their native aqueous environment. Biochemical techniques used to identify proteins complexes do not provide information about the location of the complexes within the cells and sometimes provide contradicting results. Imaging cellular components in the context of intact cells is challenging because the involved dimensions are at the nanoscale, requiring a resolution beyond stateof-the-art fluorescence microscopy, while nanoscale resolution achieved with transmission electron microscopy (TEM) involves plastic embedding or freezing, and sectioning of the cells. The length scale at which protein interactions occur is exemplified in Fig. 1 for the dimer formation of the epidermal growth factor (EGF) receptor (EGFR), a transmembrane receptor playing a critical role in the pathogenesis and progression of many different types of cancer [1]. An important question is under which conditions and in which cellular regions dimerization occurs. A biologist or biophysicist would ideally have the availability over a microscopy technique with a spatial resolution of a few nanometers to identify the protein complex subunits in the images while the cell would remain intact and in its natural aqueous environment. This example reflects one of many experiments related to the complex interplay of the approximately 20,000 proteins at work in human cells.

We are using scanning transmission electron microscopy (STEM) of cells in liquid [2, 3], so called Liquid STEM, to study protein complex subunits. Live eukaryotic cells are grown on silicon microchips with silicon nitride ( $\mathrm{SiN}$ ) membrane windows, and incubated with specific protein labels consisting, for example, of gold nanoparticles (AuNPs) (Fig. 1) or fluorescent quantum dots. The samples are then studied with STEM using either of two available modalities. On account of the atomic number (Z) contrast of the annular dark field (ADF) detector of STEM, nanoparticles of a high-Z material, such as $\mathrm{Au}-\mathrm{NPs}$, can be detected within the background signal produced by a low-Z liquid, such as water or cells. The highest resolution is obtained using STEM at $200 \mathrm{keV}$ electron beam energy, for which the cells are placed in a microfluidic chamber with electron transparent windows, see Fig. 2A. This approach can be used for either fixed or live cells [4] in saline buffer solution. Correlative fluorescence microcopy and Liquid STEM is readily possible. For certain studies, cells can also be imaged with an approach essentially not more difficult than fluorescence microscopy. Cells are fixed, transferred into deionized water, cooled to $4^{\circ} \mathrm{C}$, and their thin regions are imaged in hydrated state using environmental scanning electron microscopy (ESEM) at $30 \mathrm{kV}$ with the STEM detector, see Fig. 2B. A resolution of 4 $\mathrm{nm}$ was achieved on the Au-NPs in whole cells within the limit of radiation damage [2], and a similar resolution was obtained with ESEM on thin cellular region. Movements by Brownian motion appear to be much smaller than expected [5]. The resolution of Liquid STEM allows protein complexes in whole cells to be studied at the level of individual subunits. 
References:

[1] A. Arkhipov et al, Cell 152 (2013), p. 557.

[2] N. de Jonge et al de Jonge, Proc. Natl. Acad. Sci. 106 (2009), p. 2159.

[3] N. de Jonge and F.M. Ross, Nat. Nanotechnol 6 (2011), p. 695.

[4] D.B. Peckys et al, Biophys. J. 100 (2011), p. 2522.

[5] E.A. Ring and N. de Jonge, Micron 43 (2012), p. 1078.

[6] We thank Protochips Inc, NC, USA for providing the microchips and the Liquid STEM specimen holder. We thank E. Arzt for his support through INM, and D.W. Piston for discussions. Research in part supported by NIH grant 2R44-EB008589 (to J. Damiano).

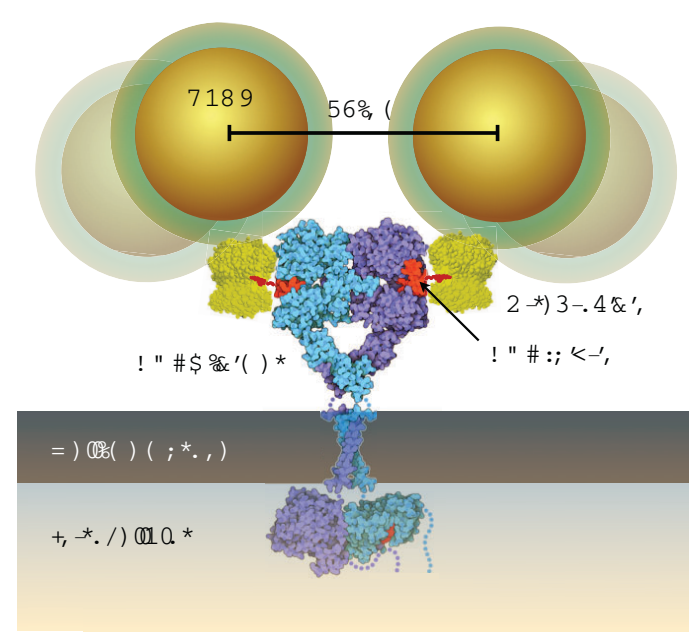

Figure 1. Molecular model of the EGFR dimer with Au-NPs conjugated via a streptavidin-biotin bond to EGF. The distance between the centers of the two AuNPs was estimated to amount to $\sim 19 \mathrm{~nm}$ for a tightly packed structure, and up to $29 \mathrm{~nm}$ when considering a rotational flexibility of the AuNPstreptavidin conjugation. The scaled drawings of streptavidin, EGF and the EGFR are derived from CPK models of the 1stp (streptavidin), 1EGF (EGF), 1NQL, 2JWA, 1M17, 1IVO and 2GS6 (EGFR) structures as found in the RCSB Protein Protein Databank, created by Jmol Version 12.2.15. Biotin size and structure was derived from the biotin model as drawn in RCSB Ligand Explorer Version 1.0.

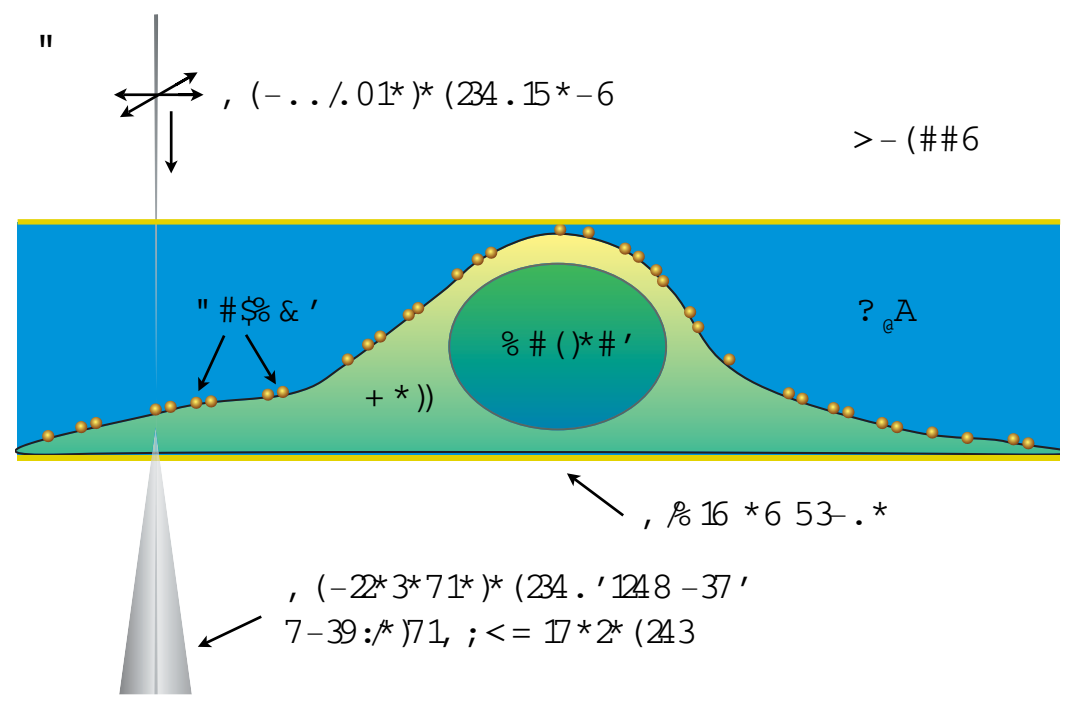

!

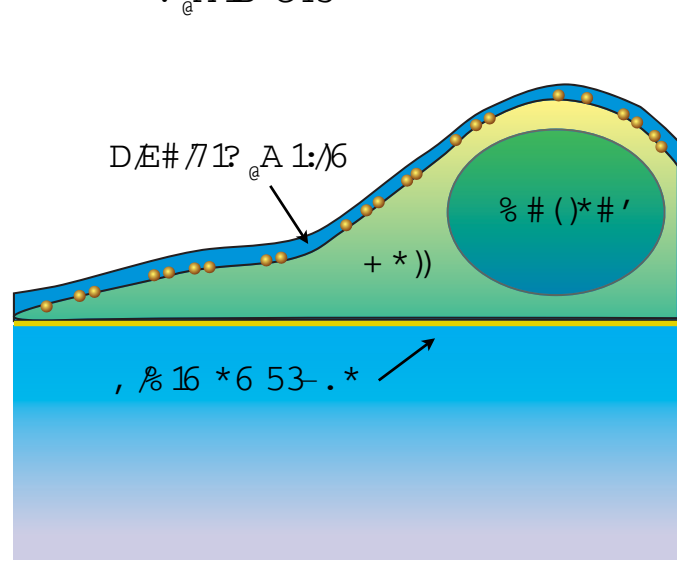

Figure 2. Principles of Liquid STEM. A whole cell is grown on a supporting SiN membrane. Proteins labeled with Au-NPs reside in the plasma membrane. Imaging is done by scanning a focused electron beam over the cell. Transmitted electrons are recorded with the STEM detector located beneath the sample. (A) The cell is fully enclosed in a microfluidic chamber with two SiN windows. (B) The cell is maintained in a saturated water vapor atmosphere, while a thin layer of water covers the cell. 